\title{
Assessment of Photon Propagation through Fatby Monte Carlo Simulation and Experimental Work in Linear Accelerator of Imam Reza Hospital
}

\author{
A.Yavar ${ }^{\mathrm{a}}$, M. F. Rahimi ${ }^{\mathrm{b}}$, I.Forouzankia ${ }^{\mathrm{a}}$, S.Mohammadi ${ }^{\mathrm{a}}$ \\ ${ }^{a}$ Department of Physics, Faculty of Science, Payame Noor University of Mashhad, Moalem 71 avenue, \\ Mashhad, post code 43391735, Iran \\ ${ }^{b}$ Department of Physics, Faculty of Science, Ferdowsi University, Azadi square, Mashhad, post code \\ 9177948974, Iran
}

\begin{abstract}
The aim of radiation therapy is to maximise the dose to the tumour, while minimising the dose to healthy tissue. Monte Carlo (MC) methods are now widely used to model radiotherapy systems for accurate treatment planning and evaluate the accuracy of the dose distributions in a variety of clinical situations involving highly heterogeneous media, such as lung or head and neck treatments. This study aimed to simulate the photon propagation in tissue using MC technique.The MC simulation was written in Fortran 77 program. Moreover, emigration of photons in tissue was determined using MCNP-4C code. The tally F5: P, $9 \mathrm{MeV}$ photons beam as a source and point detector was utilized to calculate the flux of photon at various depths of tissue. Neptun 10 PC linear accelerator of Imam Reza Hospital with $9 \mathrm{MeV}$ photon beam energy was used to determine experimental results. The result of MC methodwas evaluated withthose of MCNP-4C code and experimental result performed by clinical treatment planning system. The result of MC method shows a good consistent with those of MCNP-4C code and experimental result. The results of this research provide a method to characterize the primary radiation energy spectrum produced by a linear accelerator.
\end{abstract}

Keywords: Monte Carlo simulation, photon propagation, tissue, MCNP code, Linear accelerator of Imam Reza hospital

\section{Introduction}

Understanding the propagation and the distribution of light in biological tissue is essential for safe and effective applications of photons in medical diagnostics and therapeutics. The Monte Carlo simulation is, at present, the most accurate method for describing the coupled transport of electrons and photons in complex geometries, residual inaccuracies may nevertheless exist [1]. The Monte Carlo method can generally be described as a statistical method for numerical integration with the use of random numbers[1-7]. First descriptions of this method reach back to the year 1977 and the first popular examples were computer based calculations for radiation transport at the end of World War II [8-9]. The use of digital computers made the application of this method a valuable tool in science and technology. Generally spoken, the Monte Carlo simulation of radiation transport uses the knowledge of individual, microscopic particle interactions in matter with the corresponding probability distributions. It applies random numbers to describe the random trajectories of these particles by sampling from the underlying probability distributions. Various sampling techniques applying uniformly distributed random numbers $[0,1]$ exist $[9-10]$. The probability distributions are based on the differential cross sections for the interaction mechanisms [11].

For the energies applied in radiotherapy, photons may undergo Rayleigh scattering, photoelectric absorption, Compton scattering or production of an electron/positron pair in the electromagnetic field of atoms. The number of photons passing a certain thickness of media is decreased exponentially by these interactions. The probability for the occurrence of the single processes and the resulting change of energy and direction of created particles and the incident photon is governed by the differential cross sections. They depend on the atomic number of the medium and the energy of the photon and are based on theoretical or semi-empirical values [12]. The photoelectric effect is the dominant interaction of photons with matter, for those photons whose energy falls approximately in the range $1 \mathrm{keV}$ to $0.5 \mathrm{MeV}$. At energy around $1 \mathrm{MeV}$, Compton scattering is usually the most common interaction, because the photoelectric effect and pair production are even less likely. The probability of pair production effect rises rapidly as the photon energy increases and becomes the dominant interaction for energy more than $10 \mathrm{MeV}$ [4, 7, and 13].

Radiotherapy as an important form of cancer treatment aims at eradication of tumour cells with the use of ionizing radiation [4, 7, and 13]. A consistent quality assurance procedure is mandatory to ensure the accurate dose delivery to a tumour volume and to avoid any unnecessary harm to normal tissue. The dose, or level of radiation, is prescribed by an oncologist, and depends on the size, stage and location of the tumour, and the use 
of any other treatment modalities. The term radiotherapy does not encompass the use of non-ionising radiation to excite radiosensitisers. Tumours cannot be treated in isolation;inevitably normal tissue will also be damaged. The aim of radiation therapy is to maximise the dose to tumour, while minimising the dose to healthy tissue (reducing complication likelihood), that is, to find the greatest therapeutic ratio [14-16]. Tracing of photons propagated in tissue can be useful to achieve to this purpose. Together with the aid of a computer and MC codes, beam modelling has become valuable to provide a reference dose estimate for a planned treatment. The validation of these dose calculation algorithms is commonly performed by comparisons with measured data. The reliability of measured data sets is however very dependent on several aspects, such as the stability of the accelerator or the choice of detector and experimental set-up. These limitations may thereby restrict the number of comparison points and introduce dosimetric problems to the verification.Several studies indicate that the radiation damage is intolerable if the therapy is applied before the age of three years, as it can irremediably affect the normal brain development. Considering that high grade glioma is one of the most forms of pediatric cancer, this represents a very serious limitation to the use of standard radiotherapy methods: late effects on normal brain may significantly affect the quality of life in long-term survivors[2-7]. Objective of this research was to simulate photon propagation in tissue in order to evaluate the accurate of experimental results carried out by clinical linear accelerators in tissue, especially in challenging situations where conventional dose calculation algorithms have illustrated some limitations and it is very difficult to measure using typical clinical dosimetric procedures. This simulation was included to determine the coordinate of first interaction of photon with different energy in tissue, andexiting angle of photon from tissue with the purpose of determination the widen of photon beam during cross through from tissue.

\section{Monte Carlo simulation}

\section{Material and Methods}

Monte Carlo simulation was usedto trace photon propagationin tissue using the Fortran 77 program. Most of the conventional algorithms have been developed based on the assumption that the human body is made up of water-equivalent tissues. This assumption is roughly valid since 50-80\% of the human body is water. Atomic number of tissue (and water)is 6.46 and the photons $9 \mathrm{MeV}$ used inpresent work, therefore, the most important interactions of photons with tissue were photoelectric effect and Compton scattering.

Cross section of photoelectric effect for per atom defined as [17-18]:

$$
\sigma_{p h}=4 \alpha^{4} \sqrt{2} Z^{5} \phi_{0}\left(m_{e} c^{2} / E\right)^{7 / 2}
$$

where $\phi_{0}=8 \pi r_{e}^{2} / 3=6.651 \times 10^{-25} \mathrm{~cm}^{2} ; \alpha=1 / 137 ; c=3 \times 10^{8} \mathrm{~m} / \mathrm{s} ; m_{e}=9.1 \times 10^{-31} \mathrm{~kg} ; \mathrm{Z}$ is atomic number, and $\mathrm{E}$ is energy of photon. The cross section of Compton scattering is defined as [17-18]:

$$
\sigma_{S C}=2 \pi r_{e}^{2}\left\{\frac{1+\gamma}{\gamma^{2}}\left[\frac{2(1+\gamma)}{1+2 \gamma}-\frac{1}{\gamma} \ln (1+2 \gamma)\right]+\frac{1}{2 \gamma} \ln (1+2 \gamma)-\frac{1+3 \gamma}{(1+2 \gamma)^{2}}\right\}(2)
$$

where $r_{e}$ is electron radius and $\gamma=\frac{E}{m_{e} c^{2}}$. The total cross section of photon is determined as [2-7]:

$$
\sigma_{t o t}=\sigma_{p h}+Z \sigma_{S C}
$$

according to MC simulation, if $\frac{Z \sigma_{s c}}{\sigma_{t o t}}>\delta$, that $\delta$ is random number, therefore photon is scattered and then traversed distance and move direction of photon were calculated by [16]:

$$
\begin{aligned}
& \theta=a \cos (2 \delta-1) \\
& \phi=2 \pi \delta \\
& S=\frac{-L n(\delta)}{\mu_{t o t}}
\end{aligned}
$$

Where $\theta$ and $\phi$ are scattering angles of photon related to $\mathrm{X}$ and $\mathrm{Y}$ axes, respectively (it was assumed direction of initial photon is $\mathrm{Z}$ axis). The $\mu_{t o t}$ is total linear attenuation coefficient, $\mu_{t o t}=\frac{\rho N_{A} Z}{A} \sigma_{t o t}$ that $\rho$ is density of matter; $N_{A}$ is Avogadro number and $\mathrm{A}$ is atomic mass of matter. The energy of scattered photon can be calculated as [17-18]:

$$
E^{\prime}=\frac{E}{1+\gamma(1-\cos \theta)}
$$

where $E^{\prime}$ is energy of photon after scattering, $E$ is energy of initial photon.

Figure 1 illustrates flowchart of photon interactionswith tissue $\left(30 \times 30 \times 30 \mathrm{~cm}^{3}\right)$ using MC method. Once launched, the photon was moved a distance where it may be scattered, absorbed, or transmitted out of the tissue. 
The photon was repeatedly moved until it either escapes from or was absorbed by the tissue. If the photon escapes from the tissue, the reflection or transmission of the photon was recorded. If the photon was scattered, its scattering angle and its coordinate were recorded. If the photon was absorbed, the position of the absorption was recorded. This process is repeated until the desired number of photons (100) has been propagated. The coordinate of first interaction of photonand final scattering angle are the most important deals in radiotherapy by photon beams, which they were determined by this simulationmethod [2-5, 19-20].

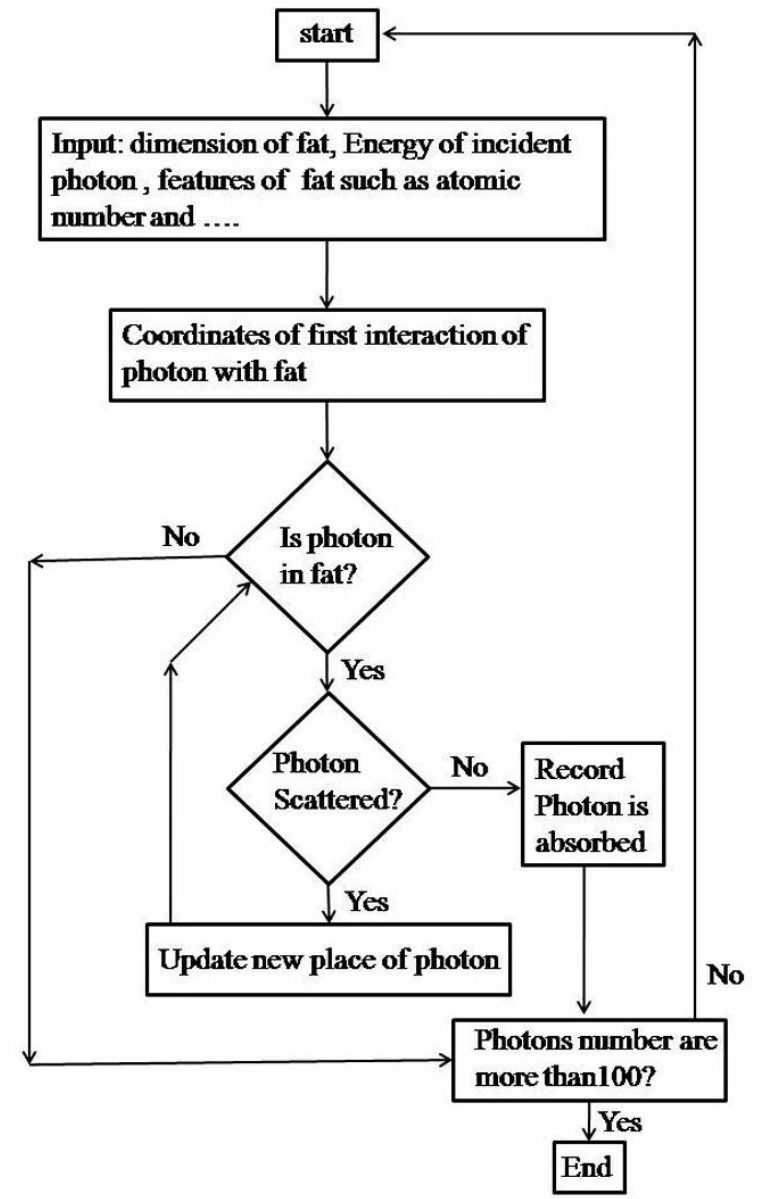

Figure 1. Flowchart of tracing of photons in fat by Monte Carlo Simulation

\section{MCNP code}

The emigration of photon beam in tissue was simulated using MCNP code. The simulation was conducted using the full continuous energy cross section available at the MCNP-4C library at $20{ }^{\circ} \mathrm{C}$ [2-3, 21]. The tally F5: $\mathrm{P}$ was used to obtain point flux of photons (particles $/ \mathrm{cm}^{2}$ ) at various depths in tissue. Photons beam with $9 \mathrm{MeV}$ energy as a photon source and point detector were used to determine the flux at each point in tissue $\left(30 \times 30 \times 30 \mathrm{~cm}^{3}\right)$ were used.

\section{Experimental work by linear accelerator of Imam Reza Hospital}

Neptun 10 PC linear accelerator with photon beam $9 \mathrm{MeV}$ at Imam Reza hospital wasemployed in thiswork. Dose measurements were carried out in a phantom consisting of water-filled tank with a scanning volume of $\left(30 \times 30 \times 30 \mathrm{~cm}^{3}\right)$ and with $\mathrm{SSD}=100 \mathrm{~cm}($ SSD is distance between accelerator to phantom). The Farmer detector type FC65-G was utilized to determine the absolute dosimetry of photon in several depths inside the phantom $(2 \mathrm{~cm}, 10 \mathrm{~cm}, 20 \mathrm{~cm}$, and $28 \mathrm{~cm})$. All farmer type chambers are designed for measurements with high reproducibility in air, in solid or in water phantom. They are suitable for absolute dosimetry of photon, electron and proton beams in radiotherapy. The absolute dosimetry is proportional with photon numbers that passed from a point in matter; therefore, experimental results were comparable with Monte Carlo results. Moreover the widening of photon beam in water phantom was determined. 


\section{Result and Discussion}

The figures 2, 3, and 4 show simulation of photon propagation in tissue using Monte Carlo simulation, MCNP code, and experimental work, respectively. In these three figures, axis $\mathrm{Y}$ was different for various methods. Axis Y wasnumber of photons, absolute dose, and point fluxin Monte Carlo method, experimental work and MCNP code, respectively. These three different quantities were proportional to each other and their differentwere not important in present work, because the coordinate of first interaction of photon in tissue and also penetration depth of photon in tissue were the most important deals. The axis $\mathrm{X}$ in these 3 figures indicatesthe penetration depth and the coordinate of first interaction of photon in tissue. As shown in figures 2 , 3 , and 4, the depth of first interaction of photon in tissue was observed in $2 \mathrm{~cm}, 2.5 \mathrm{~cm}$, and $3 \mathrm{~cm}$ using theMC method, experimental work, and MCNP code, respectively. The deviation of these results may be due to several reasons: (1) in MC method and MCNP code, a fix energy $9 \mathrm{MeV}$ was used,although in experimental work, a photonsbeam spectrum with energy of 0 to $9 \mathrm{MeV}$ was utilized. (2) InMC method and MCNP code, fat in the role oftissue was used however in experimental work, the water was utilized instead of tissue, because it was impossible to prepare tissue in $\left(30 \times 30 \times 30 \mathrm{~cm}^{3}\right)$ volume(these dimensions wereminimumneeded size of phantom toexposure by linear acceleration of Imam Reza). The water was used in role of tissue, because of its similar chemical structure and availability. (3) In MC method, only two cross sections of photoelectric effect and Compton scattering were used and other cross sections were omitted because of their diminutive, although MCNP code uses all photon interactions and also electrons produced by the interactions of the photons in tissue.
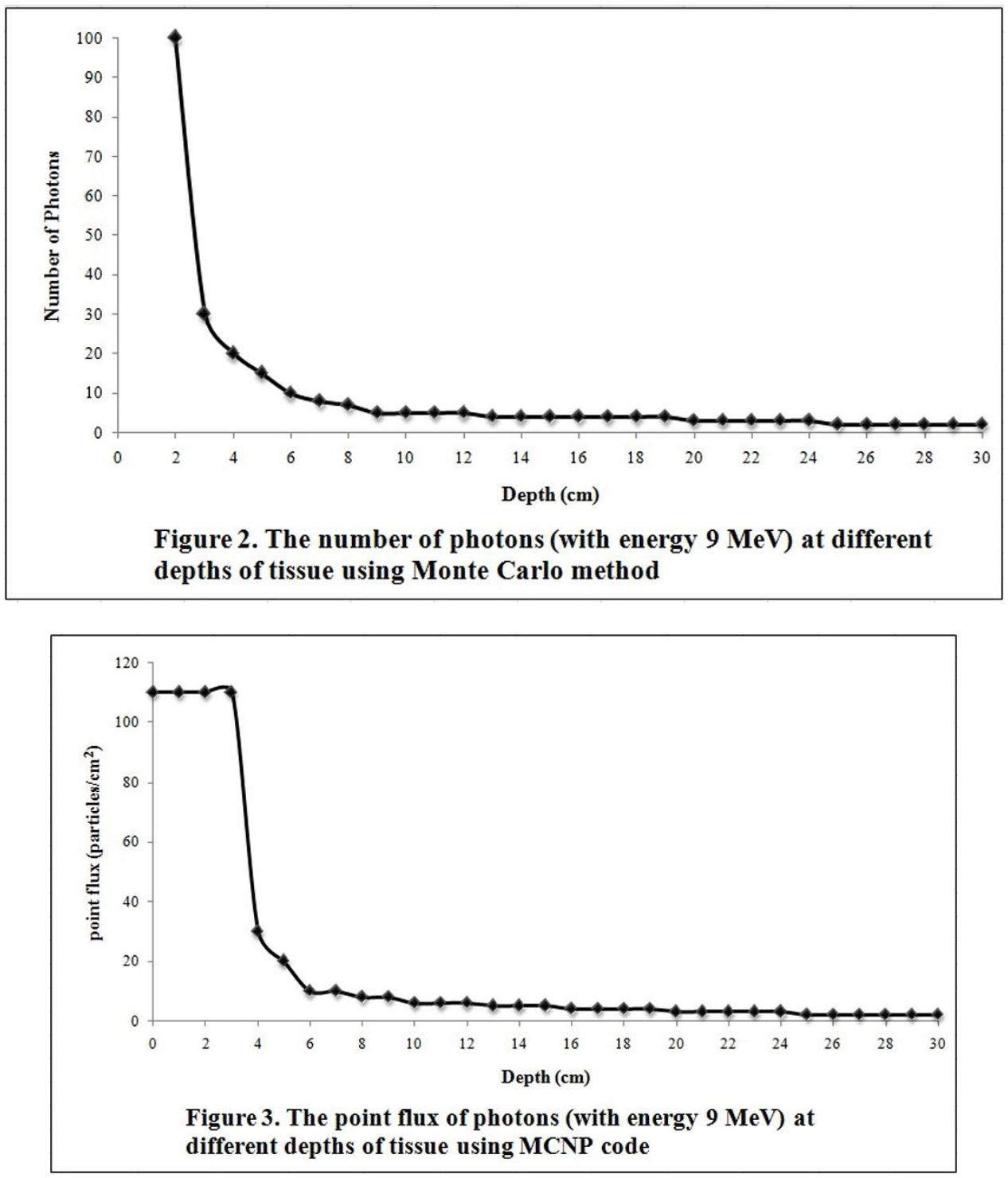


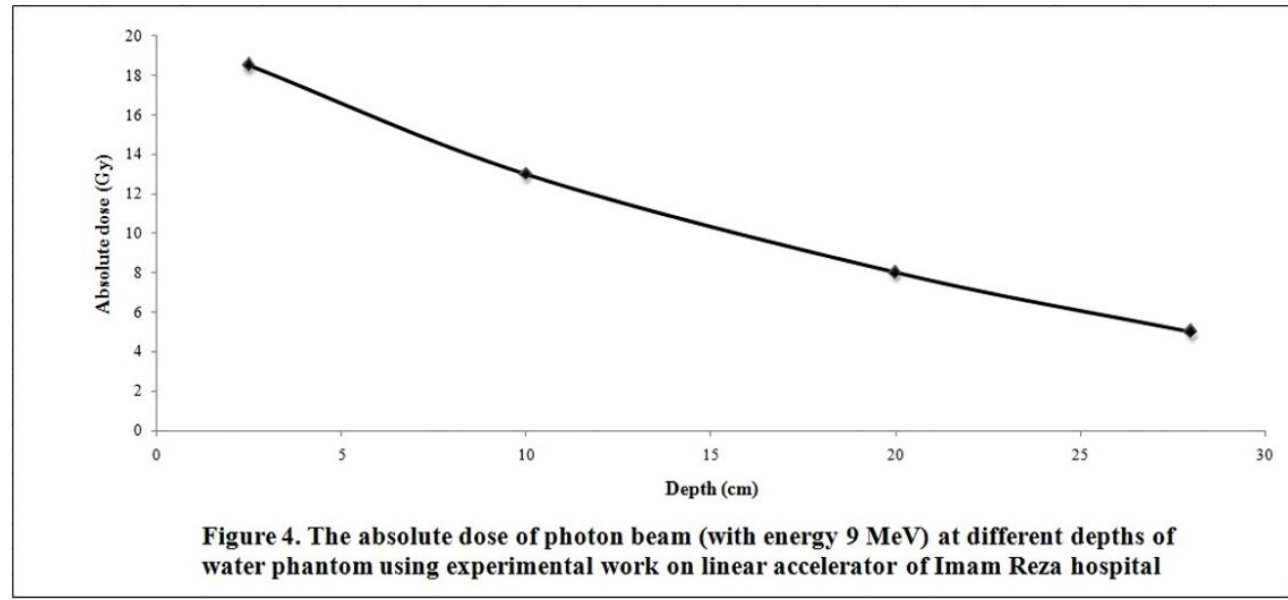

As exhibited in figure 5, primary photon beam was widened during cross through in water phantom. The exiting angle of primary photon beam after cross through in water phantom $\left(30 \times 30 \times 30 \mathrm{~cm}^{3}\right)$ was obtained around 3 degree by experimental work, which it was consistent with those of MC method 2 degree.

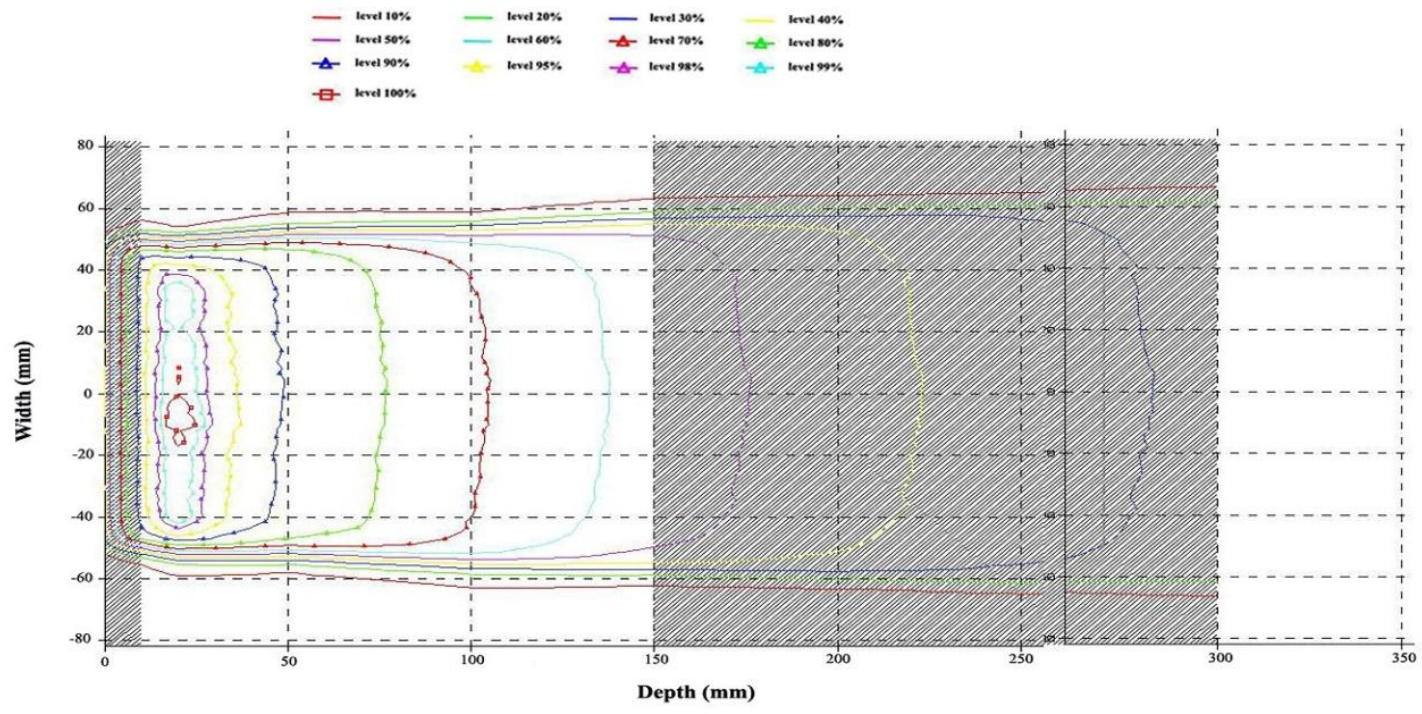

Figure 5. Determination of scattering angle of initial photon beam in crossing through the water phantom $(30 \mathrm{~cm} \times 30 \mathrm{~cm} \times 30 \mathrm{~cm})$ by experimental work at linear accelerator of Imam Reza hospital

Figure 6is illustrated coordinate of first interaction of photon in tissue versus different energy of photons. As it was expected, penetration depth of photons with lower energy was lower than others.

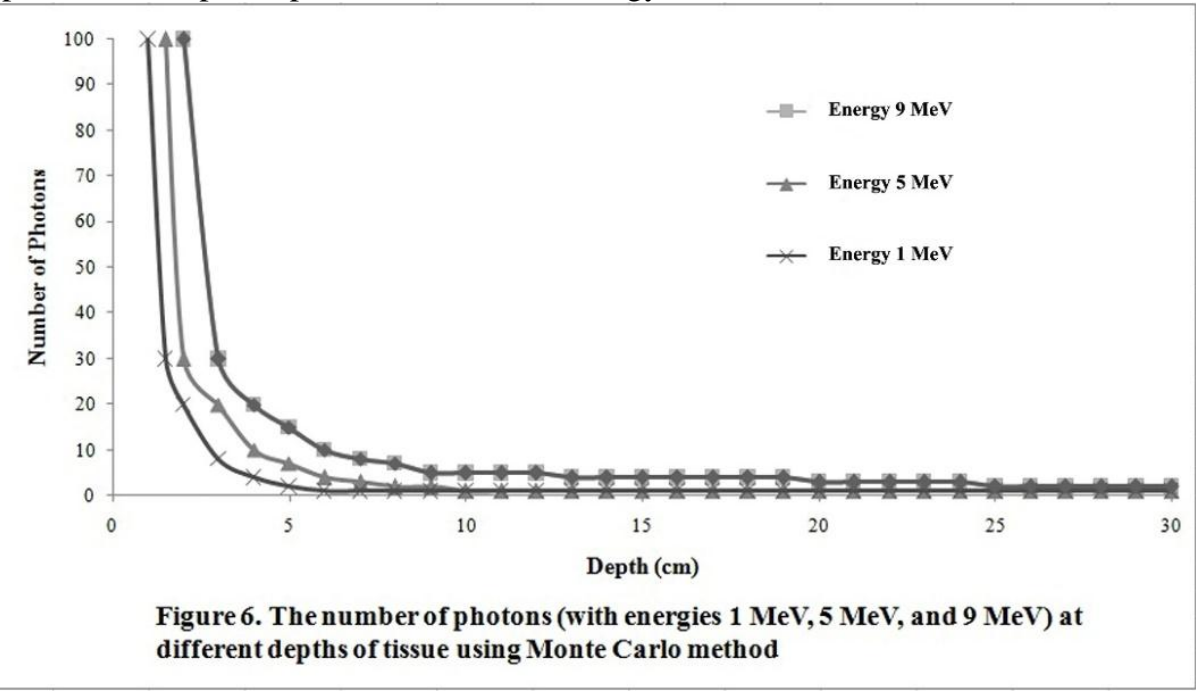




\section{Conclusion}

This work has presented a MC simulation for the transport of photon in biological tissue that explicitly supports fluorescent activity inside the tissue. This feature enables the simulation of optical homographs with complex structures, such as small animal phantoms and will provide a way to better understand photon propagation issues and thus optimize reconstruction algorithms. The result of MC method was evaluated by results of MCNP code and experimental work. The depth of first interaction of photon in tissue was determined in $2 \mathrm{~cm}, 2.5 \mathrm{~cm}$, and $3 \mathrm{cmusingMC}$ method, experimental work, and MCNP code, respectively. Moreover, the exiting angle of primary photon beam after cross through in water phantom determined by experimental work ( 3 degree) was in consistent with those of MC method ( 2 degree) in tissue. As a future work, simulation of photon propagation in multilayer material include tissue, muscle, bone can be carried out.

\section{Acknowledgments}

The present work is made possible through the help and support from group of linear accelerator of Imam Reza hospital as well as Dr.Binesh and Dr.Ariai to valuable advices in Mathematica software.

\section{References}

[1]. Zhu, C., \& Liu, Q. 2013. Review of Monte Carlo modeling of light transport in tissues. Journal of Biomedical Optics. 18(5), 050902, 1-12.

[2]. Crowe, S. B. 2011. The development of Monte Carlo techniques for the verification of radiotherapy treatments. Ph.D. thesis. Queensland University of Technology, Australia.

[3]. Katukuri, V. M., 2005. Photon transport in turbid media: simulation and experimental study. Sri Satya Sai University, India.

[4]. Moreno, M. Z. 2005. Monte Carlo simulations for dosimetric verification in photon and electron beam radiotherapy. Ph.D. thesis. Universidade Nova De Lisboa, Portugal.

[5]. Sempau, J., Reyes, A. S., Salvat, F., Tahar, H. O., Jiang, S. B., \&Varea, J. M. F. 2001. Monte Carlo simulation of electron beams from an accelerator head using PENELOPE. Journal of Physics in Medicine and Biology. 46, 1163-1186.

[6]. Sidek, M. A. J. 2010. Monte Carlo investigations of radiotherapy beams: studies of conventional, stereotactic and unflattened beams. Ph.D. thesis. The University of Birmingham, United Kingdom.

[7]. Spiga, J. 2010. Monte Carlo simulation of dose distributions for synchrotron microbeam radiation therapy. Ph.D. thesis. UniversitaDegliStudi Di Cagliari, Italy.

[8]. Eckhardt, R. 1987. Stan Ulam, John von Neumann, and the Monte Carlo Method. Los Alamos Science. 131-137.

[9]. Kalos,M.H. \& Whitlock, P.A. 2004. Monte Carlo Methods. Weinheim: Wiley VCH.

[10]. Salvat, F., Fernandez-Varea, J. M. \&Sempau, J. 2006. PENELOPE-2006: A Code Systen for Monte Carlo Simulation of Electron and Photon Transport, France: OECD Nuclear Energy Agency.

[11]. Verhaegen, F. \&Seuntjens, J. 2003. Monte Carlo modeling of external radiotherapy photon beams. Journal of Physics in Medicine and Biology. 48(21): R107.

[12]. Hubbell, J.H. 2006. Review and history of photon cross section calculations. Phys. Med. Biol. 51, R245-R262.

[13]. Elshemey, W.M., Elsayed, A.A., \& El-Lakkani, A. 1999. Physical characteristics of X-ray scattering in fat and blood. Radiation Measurements. 30(6): 715-723.

[14]. Johns, H.E. \& Cunningham, J.R. 1983. The Physics of Radiology, Fourth Edition. Charles C. Thomas, Springfield, Illinois.

[15]. Papanikolaou, N., Battista, J.J., Boyer, A.L., Kappas, C., Klein, E., Mackie, T.R., Sharpe, M. \&Dyk, J.V. 2004. Tissue Inhomogeneity Corrections for Megavoltage Photon Beams: Report of AAPM Task Group No.65, Madison, USA.

[16]. Prahl, S.A. 1988. Light Transport in Tissue. PhD thesis, University of Texas at Austin.

[17]. Leo, R.W. 1987. Techniques for Nuclear and Particle physics Experiment: A how - to approach. Springer, Verlag.

[18]. Tayal, D.C. 1982. Nuclear Physics. Himalaya Publishing House, Bombay.

[19]. Binzoni, T., Leung, T.S., Giust, R., Rüfenacht, D. \&Gandjbakhche, A. H. 2008. Light transport in tissue by 3D Monte Carlo: Influence of boundary voxelization. Computer Methods and Programs in Biomedicine, 89(1): 14-23.

[20]. Zolek, N.S., Liebert, A., \&Maniewski, R. 2006. Optimization of the Monte Carlo code for modeling of photon migration in tissue. Computer Methods and Programs in Biomedicine, 84(1): 50-57.

[21]. Briesmeister, J.F. 1997. MCNP - a General Monte Carlo N-Particle Transport Code (Version C), Oak Ridge National Lab. 